\title{
The involvement of centralized and distributed processes in sub-second time interval adaptation: an ERP investigation of apparent motion
}

\author{
Utku Kaya, ${ }^{1,2}$ (D) Fazilet Zeynep Yildirim ${ }^{3,4}$ and Hulusi Kafaligonul ${ }^{1,3}$ (D) \\ ${ }^{1}$ National Magnetic Resonance Research Center (UMRAM), Bilkent University, Ankara, Turkey \\ ${ }^{2}$ Informatics Institute, Middle East Technical University, Ankara, Turkey \\ ${ }^{3}$ Interdisciplinary Neuroscience Program, Bilkent University, Ankara, Turkey \\ ${ }^{4}$ Institute of Psychology, University of Bern, Bern, Switzerland
}

Keywords: apparent motion, auditory adaptation, event-related potentials, sub-second time intervals, visual adaptation

\begin{abstract}
Accumulating evidence suggests that the timing of brief stationary sounds affects visual motion perception. Recent studies have shown that auditory time interval can alter apparent motion perception not only through concurrent stimulation but also through brief adaptation. The adaptation after-effects for auditory time intervals was found to be similar to those for visual time intervals, suggesting the involvement of a central timing mechanism. To understand the nature of cortical processes underlying such aftereffects, we adapted observers to different time intervals using either brief sounds or visual flashes and examined the evoked activity to the subsequently presented visual apparent motion. Both auditory and visual time interval adaptation led to significant changes in the ERPs elicited by the apparent motion. However, the changes induced by each modality were in the opposite direction. Also, they mainly occurred in different time windows and clustered over distinct scalp sites. The effects of auditory time interval adaptation were centred over parietal and parieto-central electrodes while the visual adaptation effects were mostly over occipital and parieto-occipital regions. Moreover, the changes were much more salient when sounds were used during the adaptation phase. Taken together, our findings within the context of visual motion point to auditory dominance in the temporal domain and highlight the distinct nature of the sensory processes involved in auditory and visual time interval adaptation.
\end{abstract}

\section{Introduction}

Time perception is essential for survival in a dynamic world. To successfully interact with the external environment, the brain processes temporal information over a wide range of timescales from milliseconds up to several hours. A fundamental aspect of time perception is the ability to demarcate temporal intervals. To date, many different views and models have been proposed to account for time interval perception (for reviews, see Mauk \& Buonomano, 2004; Grondin, 2010; Merchant et al., 2013). Certain models posit a central common mechanism (e.g. a supramodal clock) to explain time interval perception and the corresponding neural activity. Other

Correspondence: Hulusi Kafaligonul, Aysel Sabuncu Brain Research Center, Bilkent University, Ankara 06800, Turkey.

E-mail: hulusi@bilkent.edu.tr

Received 21 June 2017, revised 22 August 2017, accepted 29 August 2017

Edited by Sophie Molholm

Reviewed by Stephan Getzmann, Leibniz Research Centre for Working Environment and Human Factors, Germany; and Jean-Paul Noel, Vanderbilt University, USA

The associated peer review process communications can be found in the online version of this article. models asserted multiple distributed mechanisms and context dependent (e.g. modality specific) processes. Several studies also suggested the existence of both a central mechanism activated by a variety of timing tasks and the neural structures that are selectively engaged by different timing contexts. Accordingly, timing models have been developed to include both central and distributed neural mechanisms by proposing that temporal interval estimation depends on the interaction between these processes (Merchant \& de Lafuente, 2014).

To understand the contribution of central and distributed processes to the perception of sub-second time intervals, audiovisual paradigms have been extensively used. The findings along this direction have highlighted the distinct temporal characteristics of the two modalities. In general, sensitivity to the time intervals marked by auditory signals is better than the time intervals marked by visual signals (Welch \& Warren, 1980; Burr et al., 2009; Grahn et al., 2011; Rammsayer et al., 2015). When both modalities are introduced together, the timing of brief sounds (i.e. auditory time intervals) has been found to drive the perceived timing of visual events (Fendrich \& Corballis, 2001; Morein-Zamir et al., 2003; Recanzone, 2003). This phenomenon is called temporal ventriloquism and is generally interpreted as a perceptual consequence of the superior 
temporal resolution of the auditory system. More importantly, it has been shown that cross-modal temporal adaptation occurs between audition and vision, such that adapting a temporal property in one modality affects the perception of that specific property introduced through the other modality (Murai et al., 2016). For instance, adapting observers to a certain auditory rate has a significant effect on the perceived rate of subsequent visual flicker, and vice versa (Levi$\tan$ et al., 2015). These results highlight sensory processes operating over two modalities and hence point to the involvement of central timing mechanisms. On the other hand, Li et al. (2015) have provided evidence that duration adaptation after-effects for audition and vision can be obtained concurrently, suggesting that modality-specific duration adaptation can operate in parallel. They also found that these concurrent after-effects of audition and vision are contingent on low-level auditory but not visual attributes. Accordingly, these findings emphasize the recruitment of different processes in the after-effects induced by each modality (see also Bruno \& Cicchini, 2016).

Auditory dominance in time percepts have been illustrated in other aspects of vision, which do not require explicit timing judgements, but which significantly depend on the timing of visual events. Several studies found that the timing of auditory signals (i.e. the time interval defined by brief static sounds) can alter the perception of visual apparent motion, presumably by altering the perceived timing of the individual frames of the apparent motion stimuli and/ or the inter-stimulus intervals (ISIs) between those frames (Getzmann, 2007; Freeman \& Driver, 2008; Kafaligonul \& Stoner, 2010, 2012; Shi et al., 2010). Moreover, using a temporal adaptation paradigm, Zhang et al. (2012) investigated the influence of time interval adaptation (auditory or visual) on motion perception. Their results showed that adapting observers to different time intervals induced significant after-effects on the perception of visual Ternus displays (i.e. apparent motion). Adaptation to auditory and visual time intervals induced after-effects in the same direction, highlighting the role of a central supramodal mechanism in the observed after-effects for motion perception.

These studies provide evidence that not only concurrent presentation of sound timing (time intervals) but also the previous experience acquired through a brief adaptation phase is also involved in shaping visual motion perception. However, the neural mechanisms underlying these adaptation after-effects remain unclear. A fundamental question is whether they are mostly mediated by a central supramodal mechanism or by distributed (modality-specific and/or cross-modal) sensory processes. In the current study, we aimed to address this question by looking for the cortical processes involved in time interval after-effects on visual motion. We adapted observers to different time intervals using brief static sounds or visual flashes and recorded EEG activity for the subsequently presented visual apparent motion (Experiment 1). To identify the contribution of central and distributed processes, we investigated the common and distinct effects of auditory and visual time interval adaptation on the ERPs across all scalp sites. We predicted that a central supramodal process would be revealed by common electrode sites and ERP components at which the after-effects (in the same direction) become significant for both modalities. On the other hand, any distinct after-effects of audition and vision on the spatiotemporal profile of the neural activity would imply the involvement of specific processes for each modality. In addition to testing, this basic hypothesis on sub-second time interval adaptation, by identifying scalp sites at which the auditory time interval after-effects take place, we were also able to shed light on how the temporal information provided by audition is used by the sensory system for visual motion processing.
Moreover, to better assess the perceptual consequences of time interval after-effects on the ERPs elicited by apparent motion, we designed a follow-up psychophysical experiment (Experiment 2). In this experiment, we used a sensitive behavioural approach to quantify the influences of time interval adaptation on perceived speed (a motion aspect which significantly depends on the time interval marked by two motion frames) and looked for common and distinct perceptual changes induced by each modality.

\section{Materials and methods}

\section{Participants}

Eighteen observers (age range: 21-32 years) participated in the main EEG experiment (Experiment 1), and eight observers (age range: 21-30 years) took part in the follow-up psychophysical experiment (Experiment 2). Two observers completed both experiments. All observers had normal or corrected-to-normal visual acuity and normal hearing. None of them had a history of neurological disorders. Participants gave informed consent, and all procedures were in accordance with international standards (Declaration of Helsinki, 1964) and approved by the ethics committee at Ankara University.

\section{Apparatus}

We used MATLAB version 7.12 (The MathWorks, Natick, MA, USA) with the PSYChtoolboX 3.0 for stimulus presentation and data acquisition (Brainard, 1997; Pelli, 1997). Visual stimuli were presented on a 20 -inch CRT monitor $(1280 \times 1024$ pixel resolution and $100 \mathrm{~Hz}$ refresh rate) at a viewing distance of $57 \mathrm{~cm}$. A SpectroCAL (Cambridge Research Systems, Rochester, Kent, UK) photometer was used for luminance calibration and gamma correction of the display. Sounds were presented via either insert earphones (Experiment 1: EARTone 3A, Etymotic Research, Village, IL, USA) or headphones (Experiment 2: Sennheiser HD 518, Sennheiser Electronic, GmbH \& Co.KG, Wedemark, Germany), and amplitudes were measured by a sound-level meter (SL-4010, Lutron Electronics, Taipei, Taiwan). Timing of auditory and visual stimuli was confirmed with a digital oscilloscope (Rigol DS 10204B, GmbH, Puchheim, Germany) connected to the computer soundcard and a photodiode (which detected visual stimulus onsets). All experiments were performed in a silent, dimly lit room.

\section{Experiment 1: EEG study}

\section{Stimuli and procedure}

A small red circle ( $0.3 \mathrm{deg}$ diameter) at the centre of the display served as a fixation target. We used two-frame apparent motion $(\mathrm{AM})$ as a test stimulus. The apparent motion consisted of two "flashed" $(50 \mathrm{~ms})$ bars $(0.4 \times 3.0 \mathrm{deg})$ centred $3 \mathrm{deg}$ above the fixation circle (Fig. 1A). The spatial displacement (i.e. centre-to-centre separation) and the inter-stimulus interval (ISI) between each flashed bar were $1 \mathrm{deg}$ and $100 \mathrm{~ms}$, respectively. Each bar was brighter $\left(107 \mathrm{~cd} / \mathrm{m}^{2}\right)$ than the grey background $\left(21 \mathrm{~cd} / \mathrm{m}^{2}\right)$. Adapting stimuli were either visual or auditory (Fig. 1B). In order to adapt observers to visual time intervals, we used the same visual bars used for the apparent motion. However, instead of having a spatial separation between them, each visual bar was flashed $(50 \mathrm{~ms})$ at the same location ( $3 \mathrm{deg}$ above the fixation circle). The time interval (ISI) between the flashed bars was either $50 \mathrm{~ms}$ (shorter time interval condition) or $350 \mathrm{~ms}$ (longer time interval condition). For auditory 
time interval adaptation, two successive static clicks (50-ms duration) were used. Each click comprised of a rectangular windowed $480 \mathrm{~Hz}$ sine-wave carrier, sampled at $44.1 \mathrm{kHz}$ with 8-bit quantization and was binaurally introduced at $75 \mathrm{~dB}$ sound pressure level (SPL). As in the visual adaptation conditions, the ISI values for shorter and longer conditions were 50 and $350 \mathrm{~ms}$, respectively.

Each trial consisted of an adaptation and a test phase (Fig. 1B). During the adaptation phase, either two auditory clicks or two flashed bars with a fixed ISI were presented sequentially. The time delay (ISI) between each consecutive presentation was $600 \mathrm{~ms}$, which was good enough to separate each repetition clearly for all adaptation conditions. At the beginning (the first trial) of each experimental block, the adaptation phase consisted of 30 repetitions of successive auditory clicks or flashed bars. For the remainder of the trials, the adaptation phase included eight repetitions. After a variable blank interval (1-2 s), during which only the fixation point was present, the test pattern (apparent motion) was displayed. The direction of motion (rightward or leftward) was randomized across trials. At the end of each trial, observers were requested to indicate, by a key press, whether the test pattern moved rightward or leftward. Observers typically responded in less than $1 \mathrm{~s}$. When the response time was shorter than $1 \mathrm{~s}$, to ensure a sufficient inter-trial interval, the next trial started $1 \mathrm{~s}$ after the offset of the apparent motion. In case the participant failed to respond within $1 \mathrm{~s}$, the trial was discarded and repeated later. Observers were instructed to fixate throughout the trial and were also asked to pay attention to both the adapting and test stimuli.

Each adaptation ( 2 modalities $\times 2$ time intervals) condition was run in a separate experimental block. In addition, observers completed an experimental block including only the test apparent motion (no adaptation condition). Instead of having an adaptation phase, each trial of this block started only with a fixation circle presented for a variable (1-3 s) duration. Then, the test apparent motion was shown and the response was recorded as in the adaptation conditions. Each experimental block consisted of 40 trials in which the response times were shorter than $1 \mathrm{~s}$. The order of these five (four adaptation and one no adaptation condition) experimental blocks were randomized across observers. Observers took a short (around 5 min) break between each block. Prior to the experimental blocks, each observer was shown examples of the test apparent motion, auditory and visual adapting stimuli.

\section{EEG data acquisition}

Electroencephalography (EEG) data were recorded with a 64-channel MR-compatible system (Brain Products, GmbH, Gilching, Germany), using sintered $\mathrm{Ag} / \mathrm{AgCl}$ passive electrodes mounted on an elastic cap (BrainCap MR, Brain Products, GmbH). The EEG caps included 63 scalp electrodes and an additional electrocardiogram (ECG) electrode attached to the back of each subject to control for cardioballistic artefacts. The placement of scalp electrodes was based on the international 10/20 system. Two of the scalp electrodes, FCz and $\mathrm{AFz}$, were the reference and ground electrodes, respectively. A syringe and q-tips were used to apply conductive paste (ABRALYT 2000, FMS, Herrsching-Breitbrunn, Germany) and to reduce impedances in each EEG channel. Electrode impedances were kept below $20 \mathrm{k} \Omega$ (typically below $10 \mathrm{k} \Omega$ ) and they were monitored throughout the experiment for reliable recording. EEG signals were digitized at a $5 \mathrm{kHz}$ sampling rate, and band-pass-filtered between 0.016 and $250 \mathrm{~Hz}$. Vision Recorder Software (Brain Products, GmbH) was used to store stimulus markers and EEG data on a secure hard disk for further analyses.

\section{EEG preprocessing and analysis}

We analysed EEG data with BrainVision Analyzer (Brain Products, $\mathrm{GmbH}$ ), the Fieldtrip toolbox (Oostenveld et al., 2011) and our own MATLAB scripts (The MathWorks). For preprocessing, EEG signals were first down sampled to $500 \mathrm{~Hz}$, and the cardioballistic artefacts were removed by the signal from ECG channel (Allen et al., 1998). Then, the data were offline rereferenced to a common average reference and filtered through a zero phase shift
A

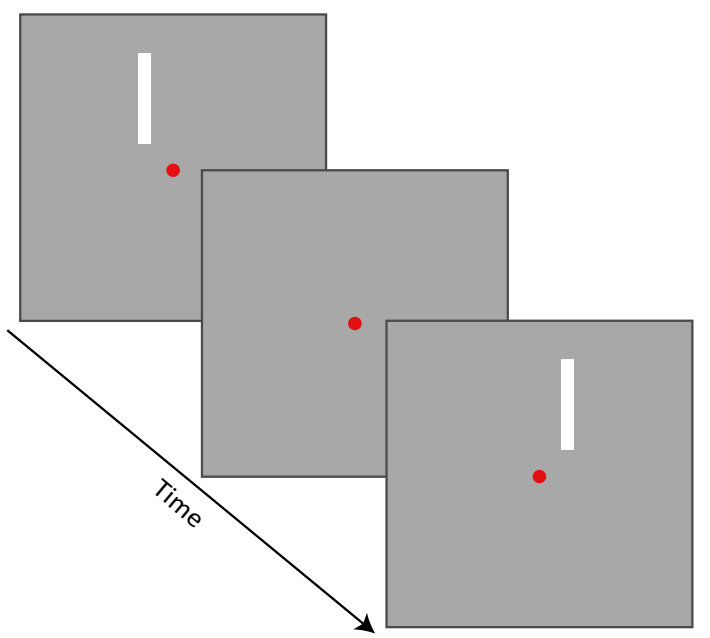

B
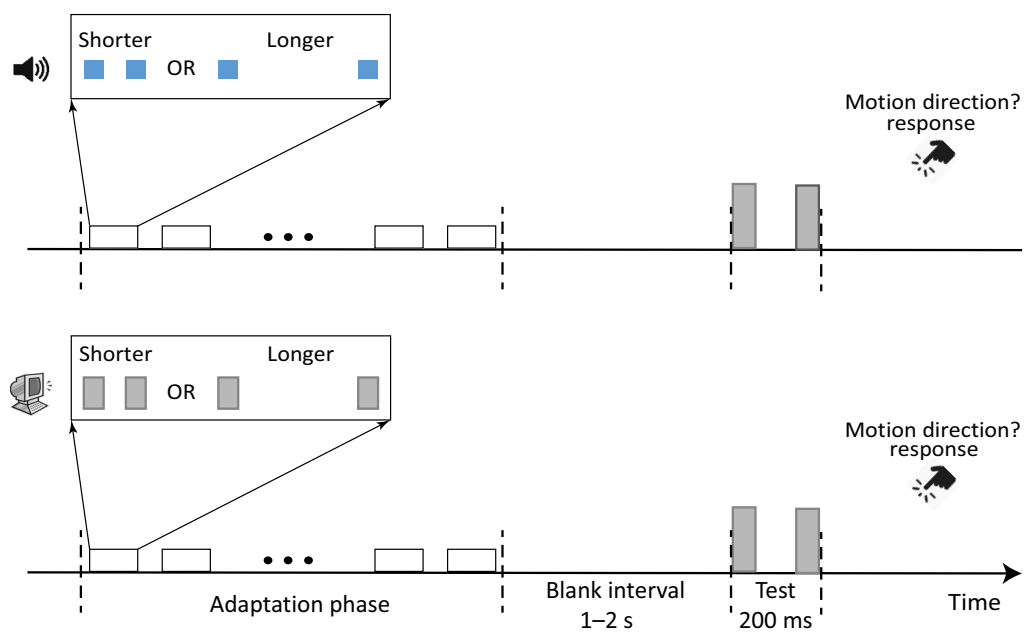

FIG. 1. Experiment 1 design. (A) The test stimulus was a two-frame apparent motion. The time interval (ISI) between each motion frame (i.e. left and right visual bars) was fixed across different adaptation conditions. (B) Each trial consisted of an adaptation and a test phase. During the adaptation phase, either two auditory clicks (blue squares) or flashed bars (grey rectangles) were presented sequentially. The time interval of these adaptors (two auditory clicks or two visual flashes) was either shorter or longer than the ISI between the motion frames. After a variable blank interval, two-frame apparent motion (test stimulus) was displayed and observers were asked to report the direction of motion. Each adaptation condition $(2$ modalities $\times 2$ time intervals) was run in separate experimental blocks. [Colour figure can be viewed at wileyonlinelibrary.com]. 
Butterworth band-pass filter $(0.5-70 \mathrm{~Hz}, 12 \mathrm{~dB} /$ octave $)$ and a 50$\mathrm{Hz}$ notch filter $(50 \mathrm{~Hz} \pm 2.5 \mathrm{~Hz}, 16$ th order $)$. After the filtering process, the data were segmented into epochs $-600 \mathrm{~ms}$ (before the onset of apparent motion) to $1200 \mathrm{~ms}$ (after the onset of apparent motion). Infomax independent component analysis was applied on these epochs in order to remove common EEG artefacts (e.g. eye blinks). Also, each trial was screened automatically by artefact rejection criteria and manually by eye. In the automatic artefact rejection, any trial with oscillations over $50 \mu \mathrm{V} / \mathrm{ms}$ or a voltage change more than $200 \mu \mathrm{V}$ was rejected. On average, $87.4 \%$ of trials were retained per condition.

After preprocessing, EEG signals from each specific electrode were averaged across trials to compute event-related potentials (ERPs) time locked to the onset of visual apparent motion. A lowpass filter (40 Hz cut-off frequency) was applied to further smooth the ERPs. Baselines were also computed from a -100 - to $0-\mathrm{ms}$ time interval prior to the onset of the apparent motion and they were subtracted from the ERPs of each condition. During the adaptation phase of our experiments, the auditory clicks were introduced through earphones, whereas the visual flashes were presented at the centre of the apparent motion path. In other words, auditory clicks and visual flashes may act as distractors and as spatial cues, respectively. Therefore, more attention may be allocated to the apparent motion subsequently presented after the visual adaptation phase than the one after the auditory phase. Such cross-modal and intramodal cueing has been found to be effective on visual cortical processing (Busse et al., 2005; Ding et al., 2014; Feng et al., 2014). To overcome these confounding factors due to the stimulation differences between two modalities and to identify adaptation after-effects specific to time intervals, we initially restricted our ERP analyses to pairwise comparisons of time interval conditions for each modality. To determine specific components and electrodes significantly affected by time interval adaptation, ERPs were compared between shorter and longer adaptation conditions using pointwise running $t$-tests (paired samples two-tailed). Furthermore, we introduced basic corrective steps to prevent false discoveries. A significant difference in conditions was defined when it was stable for at least $20 \mathrm{~ms}$ of contiguous data (i.e. 10 consecutive points at a sample rate of $500 \mathrm{~Hz}$ ) and present on at least three neighbouring electrodes meeting 0.05 alpha criterion (for the validity of this approach, see Guthrie \& Buchwald, 1991; Picton et al., 2000; Senkowski et al., 2007; Vroomen \& Stekelenburg, 2010). We also required the neighbouring electrodes to be close to the centre of major changes in the difference in ERPs (shorter-longer). Over the identified time windows and scalp sites based on the outcome of pointwise running $t$-test and corrective steps, we estimated the average ERP magnitudes for each subject. The significance of a consistent change in the average magnitudes was determined by using two-way repeated-measures ANOVAs, with the modality (auditory and visual) and time interval (shorter and longer) as factors, and follow-up pairwise comparisons (two-tailed) between shorter and longer time interval conditions.

\section{Behavioural data analysis}

For behavioural data, we only used trials included in the EEG analyses. In other words, the trials excluded during the EEG preprocessing stage were not considered for the analysis of the behavioural data. We calculated average performance across subjects for each stimulus condition. To determine whether the effects of modality and/or time interval are significant, we applied two-way repeated- measures ANOVAS and follow-up pairwise comparisons (two-tailed) to this behavioural measure.

\section{Experiment 2: Influence of time interval adaptation on perceived speed}

In a follow-up psychophysical experiment, we systematically investigated the effects of time interval adaptation on perceived speed. As detailed below, we used a sensitive speed discrimination task and our approach contained pre- and post-adaptation assessments of perceived speed.

\section{Stimuli and procedure}

The experiment consisted of pre- and post-adaptation speed measurements. In the pre-adaptation measurements, there was no adaptation phase. During each trial, a two-frame apparent motion and a comparison grating were presented with a temporal delay (ISI) of $500 \mathrm{~ms}$ (Fig. 2). The duration of each flashed bar and the ISI between them were $80 \mathrm{~ms}$ so that the physical speed of apparent motion was $6.25 \mathrm{deg} / \mathrm{s}$. Other parameters of the apparent motion were the same as those used in the main EEG experiment (Experiment 1). The comparison grating was square-wave (half-wave rectified, $1.25 \mathrm{cpd}$ ) with a $50 \%$ duty cycle. It was presented within a rectangular aperture $(6 \times 3 \mathrm{deg})$ for $360 \mathrm{~ms}$. The drifting speed was varied pseudo-randomly from trial to trial: $1,3,5,7,9,11$, 13, 15 or $17 \mathrm{deg} / \mathrm{s}$. The direction of movement (rightward or leftward) was the same for the apparent motion and the comparison grating, and they were balanced across trials. At the end of each trial, observers indicated, by pressing one of the two keys, which motion stimuli (apparent motion vs. comparison grating) appeared to move faster (two-interval forced choice). Each experimental block consisted of 180 trials $(9$ drifting speed $\times 20$ trials per stimulus), and observers completed two experimental blocks. Prior to these blocks, each observer was shown examples of visual stimuli and followed by a practice session. After collecting pre-adaptation speed data, we estimated the perceived speed of the apparent motion for each subject. A cumulative Gaussian function was fitted to the averaged data from each observer using PSIGNIFIT (version 2.5.6), a software package that implements the maximum-likelihood method described by Wichmann \& Hill (2001a,b). The 50\% point on the resultant curves yields the point of subjective equality (PSE). The PSE value is the drifting speed for which the grating was seen as faster than the two-frame apparent motion on $50 \%$ of the trials.

For the post-adaptation speed measurements, we applied a similar procedure to that of Experiment 1. Each trial consisted of an adaptation and a test phase. The adapting stimuli were either visual or auditory. To adapt observers to visual time intervals, we used the same visual bars of the apparent motion. However, they were presented at the same location ( 3 deg above the fixation) and the ISI between them could be either $40 \mathrm{~ms}$ (shorter time interval) or $240 \mathrm{~ms}$ (longer time interval). For auditory time interval adaptation, two successive static clicks (20-ms duration, $83 \mathrm{~dB}$ SPL) were used and the time intervals were the same as those used for shorter and longer visual adaptation conditions. After a blank time interval, the apparent motion and the comparison grating were shown (Fig. 2). The ISI between these motion stimuli was $500 \mathrm{~ms}$. The parameters of the apparent motion were the same as those used in the pre-adaptation measurements. The drifting speed of grating was fixed at the PSE value estimated based on the data 


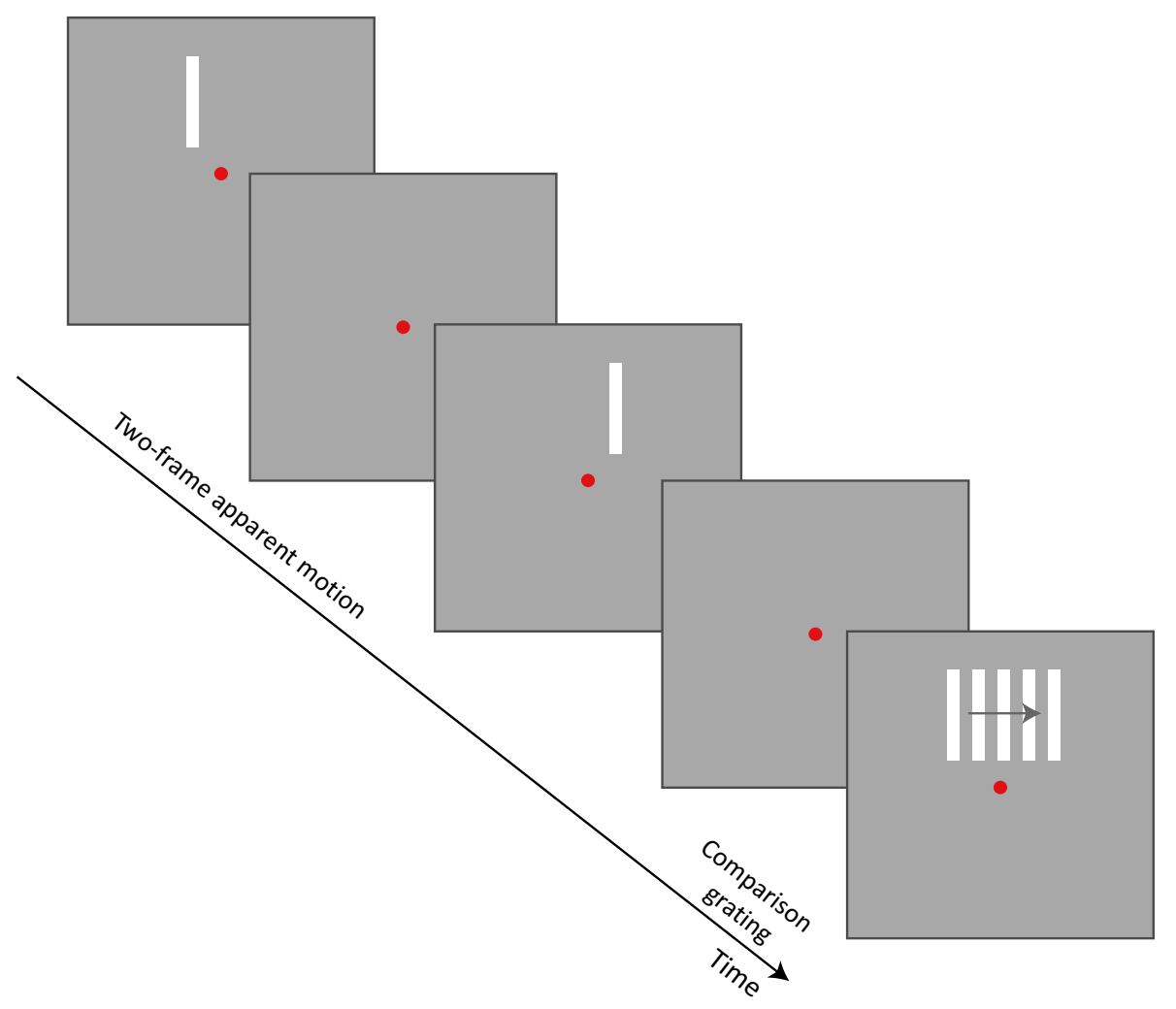

FIG. 2. Stimulus configuration for Experiment 2. To estimate the perceived speed of the apparent motion, both the apparent motion and a comparison grating were presented. The temporal delay (ISI) between the two motions was $500 \mathrm{~ms}$ and they always moved (rightward or leftward) in the same direction. At the end of each trial, observers indicated which motion stimuli appeared to move faster. [Colour figure can be viewed at wileyonlinelibrary.com].

from the pre-adaptation blocks. At the end of each trial, observers compared the speed of two motion types by a keypress. As opposed to the apparent motion, the comparison grating had more than two motion frames and there was no ISI between them. In other words, the grating did not include a time interval marked by only two motion frames and between the ISIs used in the adaptation phase. Therefore, we reasoned that any time interval aftereffects on the apparent motion should be more influential compared to the grating. Each adaptation block consisted of 20 trials, and each observer completed two blocks for each adaptation condition. Except for these changes introduced in the stimuli and procedure, all other stimulus parameters, experimental procedures and statistical analyses on the behavioural data were the same as those used in Experiment 1.

\section{Results}

\section{Experiment 1: EEG study}

\section{Behavioural results}

Figure 3 shows the average performance on the motion discrimination for each condition. As expected, the motion direction discrimination performance was well above threshold and all observers reported the direction of apparent motion with more than $95 \%$ accuracy for all conditions. A two-way repeated-measures ANOVA on the accuracy scores, with modality (auditory and visual) and adaptation interval (shorter and longer) as factors, revealed a marginally significant modality effect $\left(F_{1,17}=4.250\right.$, $\left.P=0.054, \eta_{p}^{2}=0.200\right)$. The effect of time interval $\left(F_{1,17}=1.062\right.$, $\left.P=0.317, \quad \eta_{p}^{2}=0.059\right)$ and its interaction with modality

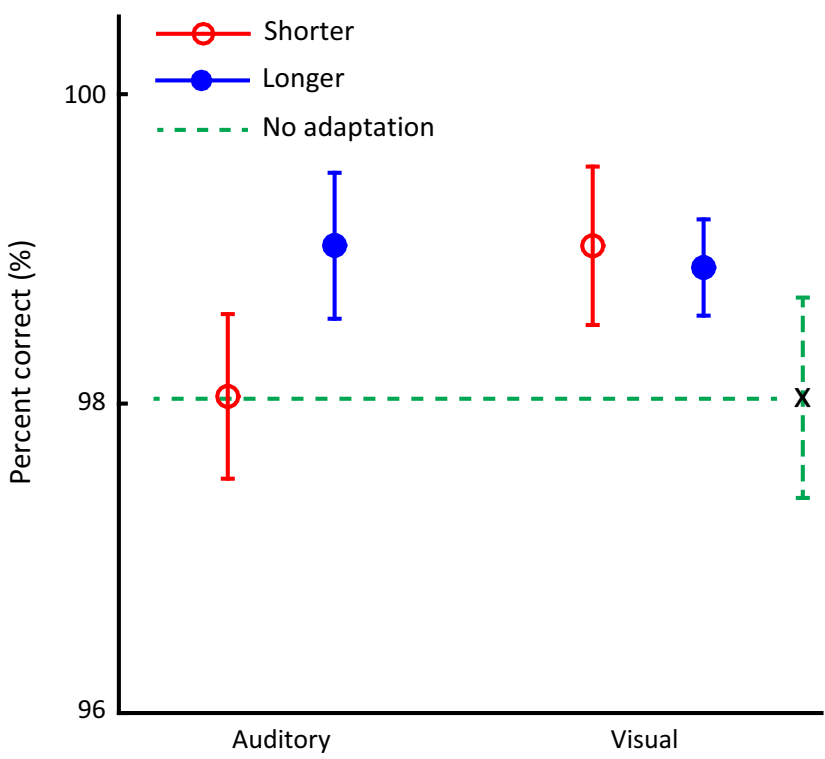

FIG. 3. Behavioural results on motion direction discrimination. Mean percentage correct values averaged across subjects $(N=18)$. The open and filled symbols correspond to shorter and longer time interval conditions, respectively. Error bars correspond to \pm SEM. The dotted line indicates the mean value for the no adaptation condition, and the error bar placed over the symbol at the end of this line represents \pm SEM. [Colour figure can be viewed at wileyonlinelibrary.com].

$\left(F_{1,17}=2.693, P=0.119, \eta_{p}^{2}=0.137\right)$ were not significant. The planned contrasts revealed only a marginally significant difference between the shorter and longer conditions of auditory adaptation 

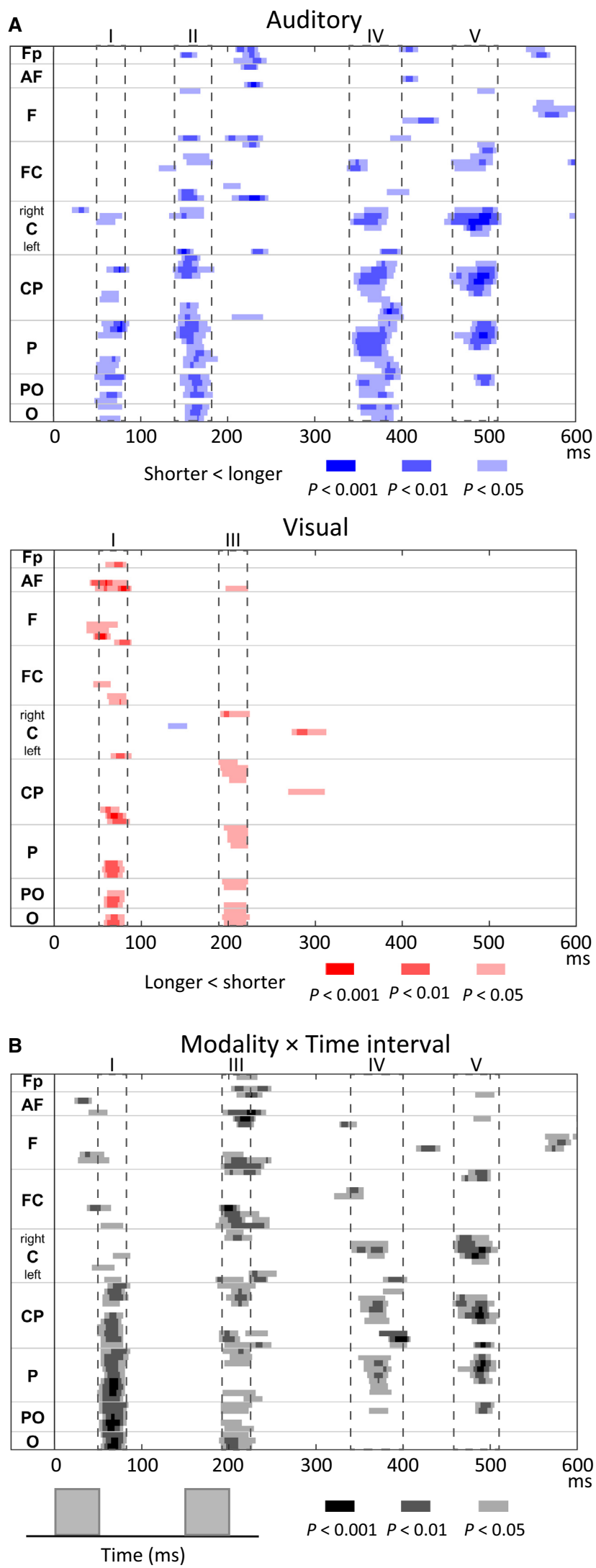

(auditory $t_{17}=-2.029, \quad P=0.068, \quad$ visual $\quad t_{17}=-1.026$, $P=0.804)$. None of the adaptation conditions were significantly different than the no adaptation condition (Bonferroni-corrected paired $t$-tests).
FIG. 4. Time courses of time interval after-effects on the evoked activity. (A) Pointwise running $t$-tests on the evoked activity to apparent motion presented after auditory and visual adaptors. The pairwise (shorter vs. longer) comparisons for each modality are shown in separate plots (auditory: upper panel, visual: lower panel). In each plot, time is displayed on the abscissa from 0 to $600 \mathrm{~ms}$ (relative to the apparent motion onset), and electrodes are displayed on the ordinate. The significant difference between time interval conditions is marked on the temporal axis by different levels. A specific time point was shaded only if at least $20 \mathrm{~ms}$ of contiguous data was significantly different (see Methods). Based on the sign of the difference between shorter and longer time interval conditions, either blue (shorter $<$ longer) or red (shorter $>$ longer) colour was used for shading. The identified time windows for further analysis are shown by the dashed rectangles (I: 50-80 ms, II: 140-180; III: 190-220 ms; IV: 340-400 ms; V: 460-510 ms). (B) Running repeated-measures ANOVAS with modality and time interval as factors. A specific time point was shaded only if at least $20 \mathrm{~ms}$ of contiguous data had significant two-way interaction (modality $\times$ time interval). Apparent motion frames are represented by grey rectangles at the bottom. The timing and durations of apparent motion frames are indicated by the position and thickness of these rectangles, respectively. Other conventions are the same as those in the upper plots. [Colour figure can be viewed at wileyonlinelibrary. com].

Time interval after-effects on the ERPs elicited by apparent motion: Time course and scalp topographies

We wanted to determine whether adaptation to distinct time intervals could affect evoked activity to visual apparent motion (i.e. the test stimulus which is exactly the same across all conditions). If so, we should observe significant differences between the ERPs for shorter and longer interval adaptation conditions. Moreover, we reasoned that if the observed differences are due to a central (supramodal) timing mechanism, the changes in ERPs for both modalities should occur around similar times and electrode locations. Otherwise, we should observe specific changes for auditory and visual adaptation conditions. To test these possibilities, we performed pairwise comparisons (pointwise running $t$-tests with basic corrective steps) between shorter and longer ERPs across all individual electrode locations for each modality (auditory or visual) separately. Comparison of shorter and longer adaptation conditions of each modality separately also allowed us to limit potential confounding factors and to focus on adaptation after-effects specific to time intervals (see Methods). For both modalities, the pairwise comparisons revealed significant differences between shorter and longer time interval conditions. However, these after-effects were in the opposite direction. As shown by Fig. 4, we identified five different time windows in which the adaptation after-effects take place. Within four of these identified time windows, we found significant two-way interactions between modality and time interval. Except for a very early component $(\mathrm{C} 1)$, the after-effects induced by auditory and visual time intervals occurred in different time windows and mostly clustered over distinct sites (Fig. 5).

In particular, the difference between the two time interval conditions was much more salient when auditory clicks were used during the adaptation phase. These differences were present within four different time windows. The average activity within $50-80 \mathrm{~ms}$ was negative and positive over parieto-occipital electrodes for the shorter and longer auditory time intervals, respectively. This significant difference was present at both hemispheres and spread over neighbouring occipital and parietal sites (Fig. 5A). As the presentation of the second visual frame defines the time interval and apparent motion, the significant changes in ERP components after the onset of the second flash $(150 \mathrm{~ms})$ are particularly important in the current study. For the negative activity within 140-180 ms (N1 component), the ERPs for the shorter auditory time interval were significantly larger in amplitude than those for the longer one. This significant effect of 
A
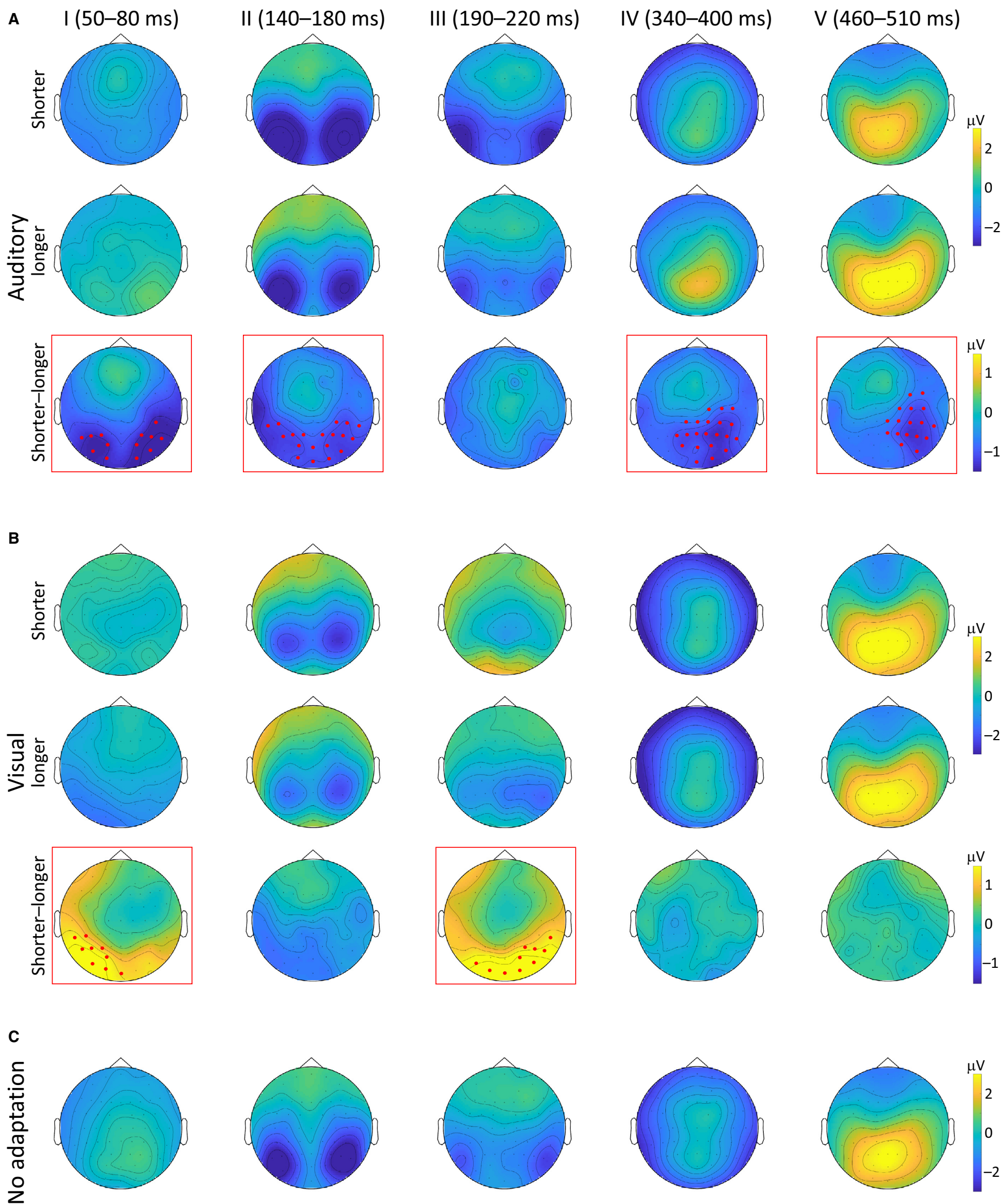

FIG. 5. Scalp topographies. Voltage topographical maps of the grand averaged ERPs within each identified time window are displayed in separate columns. Each adaptation condition and the difference maps (shorter-longer) are shown in separate rows. The significant scalp sites (see also Methods for additional criteria for significance) are marked by filled circles on the difference maps. (A) Auditory conditions, (B) Visual conditions, (C) No adaptation condition. [Colour figure can be viewed at wileyonlinelibrary.com]. 
time interval was centred at parietal regions and also existed at neighbouring parieto-occipital and parieto-central electrodes. In addition, the effects of time interval adaptation within this time range were more salient in the right hemisphere, but also present in the left hemisphere. For auditory adaptation, significant differences between the two time interval conditions were also found for the components beyond $300 \mathrm{~ms}$. The activity for the shorter time interval was less positive compared with the longer interval. As indicated by the topographic maps in Fig. 5A, the differential effects of adaptor time interval (shorter vs. longer) were centred over the parietal electrodes (and also extended over the occipital and central regions) for the 340-400 ms range. The centre of the cluster was medial relative to significant electrode locations for the 140 - to 180 -ms time window. For the 460-510 ms range, the significant differences were observed over centro-parietal sites at the right hemisphere.

Compared with the differences between auditory shorter and longer conditions, the effects of visual time interval adaptation were less salient and occurred only in two time windows. We mainly observed differences between the two visual conditions in the 50- to $80-\mathrm{ms}$ and the $190-$ to $220-\mathrm{ms}$ time windows. The activity of the longer condition was more negative than that of shorter condition within $50-80 \mathrm{~ms}$. The after-effects were clustered over parieto-occipital and parietal electrodes at the left hemisphere. In the 190- to 220-ms time range, over the occipital and parieto-occipital electrodes, the activity for the shorter condition was more positive than that for the longer condition (Fig. 5B).

Time interval after-effects on the ERPs elicited by apparent motion: Averaged ERP amplitudes from exemplar sites

Although the significant after-effects of auditory time interval led to different clusters for each time window, three electrodes (P4, P6 and CP6) were common across the four identified time windows. The grand averaged ERPs of these electrodes, where the effects of auditory time interval adaptation were commonly observed, are shown in Fig. 6A. We also computed average potentials within the identified time windows for these exemplar sites and these values are indicated by Fig. 6B. Regarding the 50- to $80-\mathrm{ms}$, 340- to $400-\mathrm{ms}$ and 460 - to $510-\mathrm{ms}$ time ranges, there was a significant difference between the auditory adaptors, but not between the visual adaptors. This is confirmed by the ANOVA tests indicating a significant interaction between modality and time interval (Table 1). Post hoc pairwise comparisons indicated that the effect of time interval was only significant for auditory conditions $\left(50-80 \mathrm{~ms}: t_{17}=2.791\right.$, $P=0.012 ; \quad 340-400 \mathrm{~ms}: \quad t_{17}=3.120, \quad P=0.006 ; \quad 460-510 \mathrm{~ms}$ : $\left.t_{17}=3.435, P=0.003\right)$, such that the longer time interval yielded higher potentials than the shorter interval. In the 140-180 ms range, a two-way repeated-measures ANOVA revealed significant main effects of modality and time interval on the ERP amplitudes; however, the interaction was not significant. The average potential for the shorter time interval was smaller than the longer interval, and the potentials for the visual stimuli were higher than for the auditory stimuli.

Similarly, we identified three neighbouring electrodes $(\mathrm{O} 1, \mathrm{Oz}$, PO7) commonly affected by visual time intervals. The grand averaged ERPs of these electrodes and the averaged potentials within the two time windows (50-80 ms and 190-220 ms) are shown in Fig. 7. For both time windows, a repeated-measures ANOVA on the average potentials revealed a significant interaction between modality and time interval (Table 1 ). In the $50-80 \mathrm{~ms}$ range, the average potentials of the shorter were significantly higher than those of the longer time intervals for vision $\left(t_{17}=-3.109\right.$,
$P=0.006)$. On the other hand, the effect of auditory time interval was in the opposite direction $\left(t_{17}=2.702, P=0.015\right)$. Post hoc pairwise comparisons on the averaged potentials within 190-220 ms revealed the shorter time interval to yield higher voltages than the longer time interval for the visual adaptors $\left(t_{17}=-2.585\right.$, $P=0.019)$, but the difference was not significant for the auditory adaptors $\left(t_{17}=1.446, P=0.166\right.$; Fig. $7 \mathrm{~B}$, lower panel). The results of additional statistical tests comparing a specific adaptation condition with the no adaptation one for both exemplar sites are presented in Table $\mathrm{S} 1$.

To understand the time course and progression of these time interval after-effects within an adaptation block, we also estimated averaged ERP amplitudes using the first (early) and the last (late) 10 trials of each block. To test the difference between the amplitudes of these subset (early vs. late) of trials, a three-way repeated-measures ANOVA including subset, modality and time interval as factors was performed. These analyses revealed only a significant effect of subset over auditory sites (P4, P6 and CP6) within 140-180 ms (Table S2). For this time range, the main effect of subset $\left(F_{1,17}=4.934, \quad P=0.040, \quad \eta_{p}^{2}=0.225\right)$ and its interaction with modality $\left(F_{1,17}=10.618, P=0.005, \eta_{p}^{2}=0.384\right)$ were significant. Compared with early trials, visual adaptation conditions had higher potentials in the late trials. Moreover, the difference between two auditory adaptation conditions became greater in these trials (Fig. S1).

\section{Experiment 2: Influence of time interval adaptation on perceived speed}

In our EEG experiment, observers performed a simple direction discrimination on two-frame apparent motion. This simple task was useful to draw attention to the test stimulus and to focus observers on a motion feature. However, the average performance values were close to $100 \%$ and they did not change significantly across the different adaptation conditions. That is to say, the significant changes in the evoked activity were not reflected in the behavioural data. We consider that this is due to the fact that the apparent motion stimulus used was supra-threshold in terms of direction discrimination and observers might have used some other cues (e.g. positional cues) for motion direction. A simple direction discrimination task with this stimulus may not be sufficient to reveal possible changes in the perception of apparent motion such as its categorization (Getzmann, 2007) and speed (Kafaligonul \& Stoner, 2010). Accordingly, we designed a psychophysical experiment to investigate the effects of time interval adaptation on perceived speed.

Our approach included pre- and post-adaptation assessments of speed. In the pre-adaptation speed measurements, we estimated the perceived speed of apparent motion through a comparison drifting grating. During the post-adaptation speed judgements, the drifting speed of grating was fixed at the point of subjective equality $(\% 50$ baseline level) estimated from each observer in the pre-adaptation sessions. If time interval adaptation does indeed affect the perceived speed of the subsequently presented apparent motion, the percentage of the trials in which apparent motion seen as faster should significantly differ between shorter and longer adaptation conditions. Otherwise, we would not expect a significant change in the percentage values. The percentage values for the post-adaptation judgements are shown in Fig. 8. We performed a two-way repeatedmeasures ANOVA on these results. The main effects of modality $\left(F_{1,7}=1.612, \quad P=0.244, \quad \eta_{p}^{2}=0.187\right) \quad$ and time interval $\left(F_{1,7}=0.946, P=0.363, \eta_{p}^{2}=0.119\right)$ were not significant. However, the two-way interaction was found to be significant $\left(F_{1,7}=11.571\right.$, 

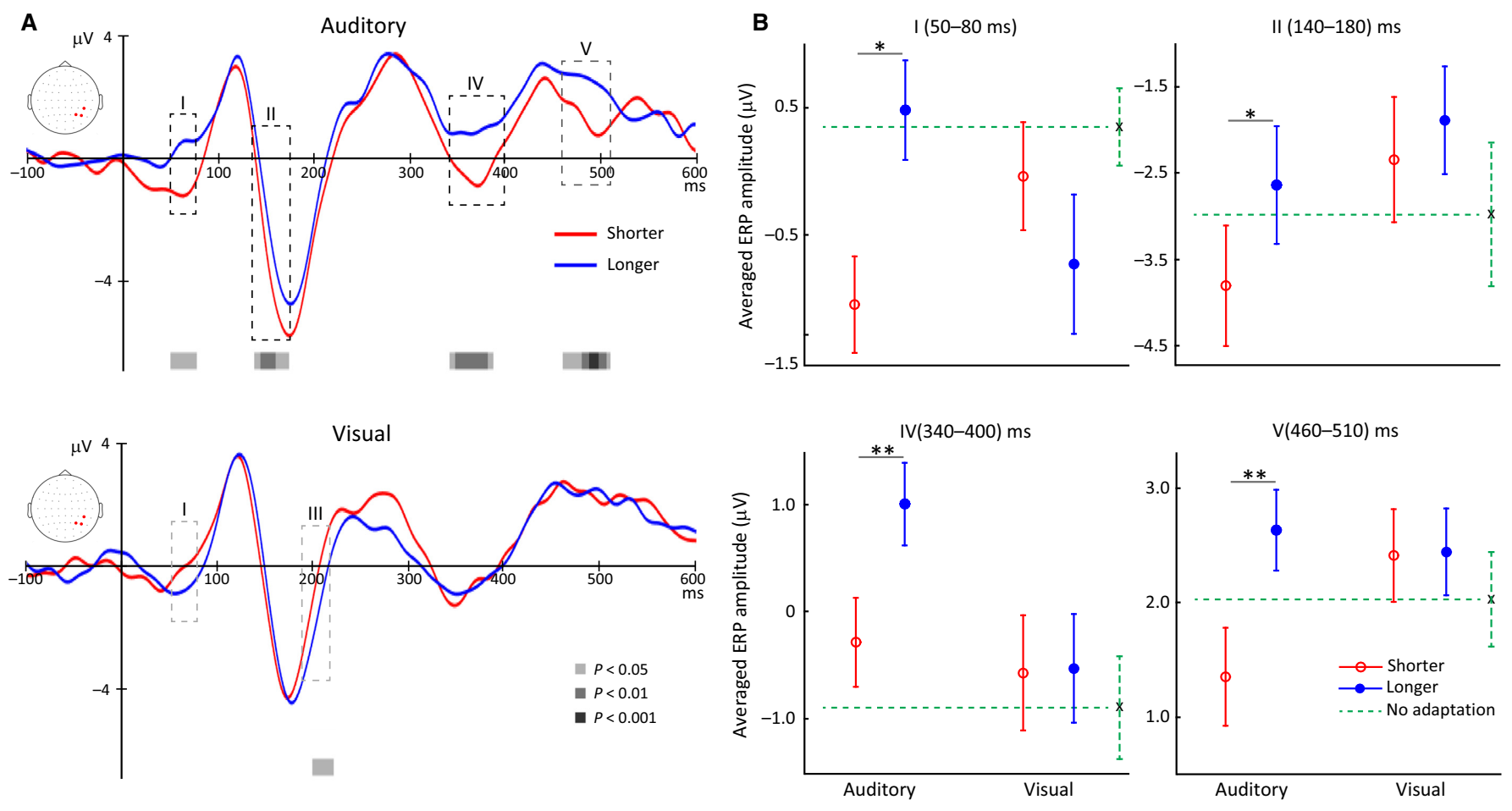

FIG. 6. Averaged activity across three common electrodes (P4, P6, CP6) where the auditory time interval adaptation had significant after-effects on the ERP components. (A) Grand averaged ERPs for different adaptation conditions. The ERPs for auditory and visual conditions are shown in separate plots (auditory: upper panel, visual: lower panel). In each plot, time-domain waveforms for shorter and longer adaptation conditions are displayed. The significant (pointwise running $t$-test, $P<0.05$ ) difference between time interval conditions are marked on the temporal axis by different grey levels. A specific time point was shaded only if at least $20 \mathrm{~ms}$ of contiguous data was significantly different (see Methods). (B) Averaged ERP amplitudes in different time windows for different adaptation conditions $(N=18)$. The temporal location of the time windows is shown in the left panel. The values for each time window are displayed in separate plots. In each plot, the open and filled symbols correspond to shorter and longer time interval conditions, respectively. Error bars indicate standard error ( \pm SEM) across subjects. The dotted line indicates the mean value for the no adaptation condition, and the error bar placed over the symbol at the end of this line represents standard error across subjects. A significant difference between shorter and longer conditions was marked with asterisk signs (paired $t$-test, $\left.* P<0.05,{ }^{* *} P<0.01\right)$. [Colour figure can be viewed at wileyonlinelibrary.com].

TABLE 1. Two-way repeated-measures ANOva on the averaged ERP amplitudes. The table summarizes the results of ANova tests on the data shown in Figs $6 \mathrm{~B}$ and 7B. The values for each time window are shown in separate rows. The upper four rows and lower two rows represent the test results for auditory (Fig. 6B) and visual sites (Fig. 7B), respectively. Significant $P$ values $(P<0.05)$ are highlighted in bold

\begin{tabular}{|c|c|c|c|c|c|c|c|c|c|}
\hline & \multicolumn{3}{|c|}{ Modality } & \multicolumn{3}{|c|}{ Time interval } & \multicolumn{3}{|c|}{ Modality $\times$ Time interval } \\
\hline & $F_{1,17}$ & $P$ & $\eta_{p}^{2}$ & $F_{1,17}$ & $P$ & $\eta_{p}^{2}$ & $F_{1,17}$ & $P$ & $\eta_{p}^{2}$ \\
\hline $50-80 \mathrm{~ms}$ & 0.029 & 0.866 & 0.002 & 1.138 & 0.301 & 0.063 & 10.661 & 0.005 & 0.385 \\
\hline $140-180 \mathrm{~ms}$ & 7.729 & 0.013 & 0.313 & 4.809 & 0.043 & 0.221 & 1.343 & 0.262 & 0.073 \\
\hline $340-400 \mathrm{~ms}$ & 7.570 & 0.014 & 0.308 & 4.444 & 0.050 & 0.207 & 5.398 & $\mathbf{0 . 0 3 3}$ & 0.241 \\
\hline $460-510 \mathrm{~ms}$ & 1.794 & 0.198 & 0.095 & 6.796 & 0.018 & 0.286 & 9.997 & 0.006 & 0.370 \\
\hline $50-80 \mathrm{~ms}$ & 0.024 & 0.879 & 0.001 & 0.017 & 0.897 & 0.001 & 14.988 & 0.001 & 0.469 \\
\hline $190-220 \mathrm{~ms}$ & 10.522 & 0.005 & 0.382 & 1.976 & 0.178 & 0.104 & 8.487 & 0.010 & 0.333 \\
\hline
\end{tabular}

$\left.P=0.011, \eta_{p}^{2}=0.623\right)$. To disentangle the source of this interaction, we performed post hoc pairwise comparisons for each modality. For auditory adaptation, the percentage values for the shorter were significantly higher than those for the longer condition $\left(t_{7}=2.465\right.$, $P=0.043$ ). Based on the speed of comparison grating, the apparent motion presented after the shorter time intervals was perceived as faster than the one presented after longer time intervals. On the other hand, the average percentage values for both visual conditions were below the baseline level and there was no significant difference between these time interval conditions $\left(t_{7}=-0.914, P=0.391\right)$.
In line with our ERP findings in Experiment 1, the behavioural results on perceived speed highlight distinct characteristics for each modality. Compared with visual time intervals, the auditory intervals induced stronger and significant adaptation effects on perceived speed. Any non-significant trend for visual time intervals points to an effect in the opposite direction. Moreover, besides providing supporting behavioural evidence to the EEG data, the psychophysical results in Fig. 8 indicate for the first time that adaptation to auditory time intervals can alter perceived speed of visual motion. 

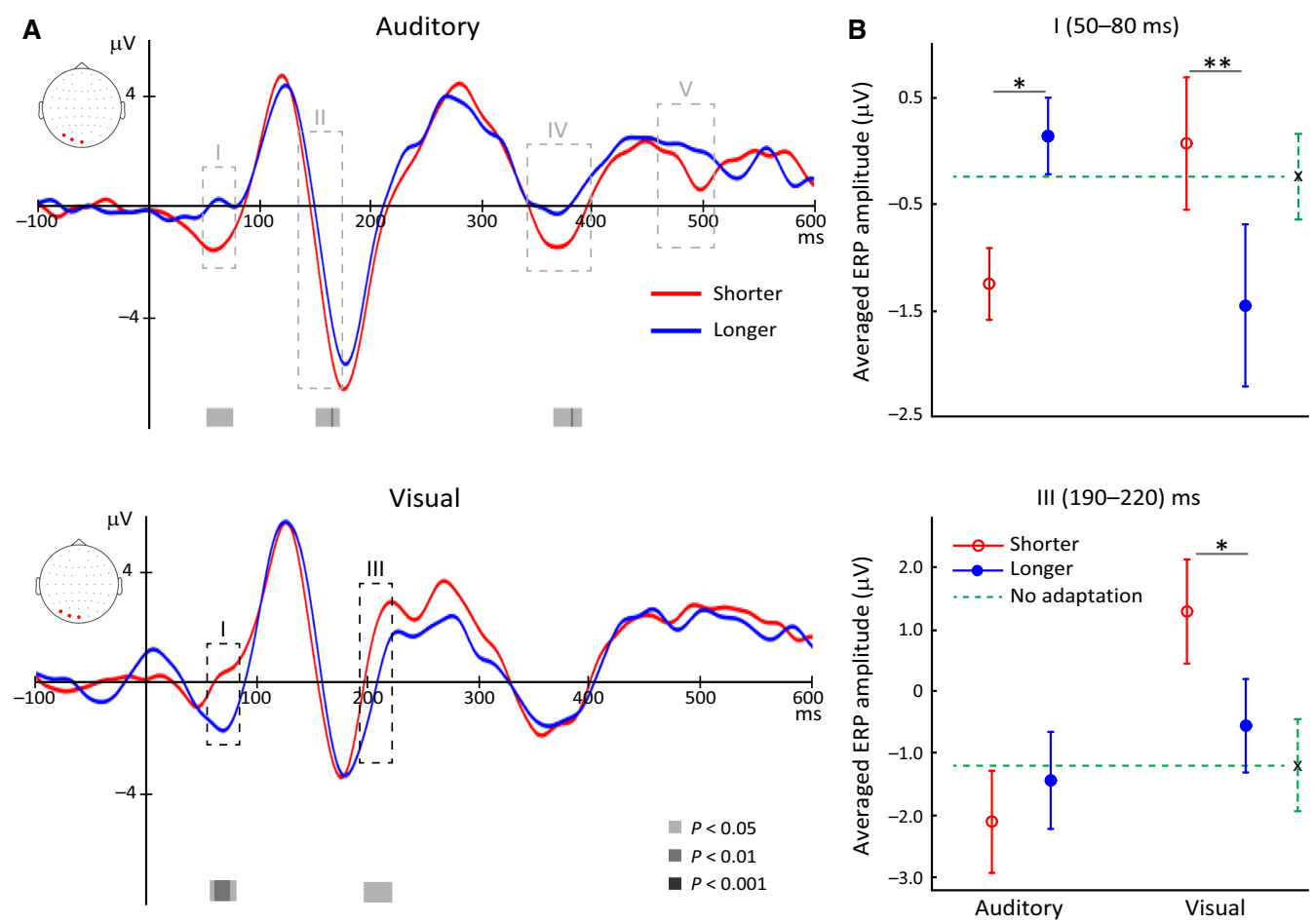

FIG. 7. Averaged activity across three common electrodes (O1, Oz, PO7) where the visual time interval adaptation had significant after-effects on the ERP components. (A) Grand averaged ERPs for different adaptation conditions. (B) Averaged ERP amplitudes in different time windows for different adaptation conditions $(N=18)$. Other conventions are the same as those in Fig. 6. [Colour figure can be viewed at wileyonlinelibrary.com].

\section{Discussion}

In the present study, we systematically examined the evoked activity to visual apparent motion under different time interval adaptation conditions. To define a specific time interval in the motion stimulus, we used two-frame apparent motion with a fixed time interval between the motion frames. Our study revealed a number of interesting and novel findings. Based on the modality (auditory clicks or visual flashes) and the time interval (shorter or longer than that of the apparent motion) used in the adaptation phase, we found significant and distinct changes in the ERPs elicited by exactly the same visual motion. First, compared with visual time intervals, the aftereffects by auditory time intervals were more salient and in the opposite direction. Second, except for changes in a very early component, the after-effects by auditory and visual adaptors occurred within different time windows. Finally, the auditory and visual adaptors led to significant changes over cluster of electrodes mostly centred at different sites. When auditory clicks were used as the adaptors, the difference between shorter and longer conditions were mostly over parietal and parieto-central regions. The significant differences between shorter and longer visual time intervals centred over occipital and parieto-occipital regions. We also observed significant after-effects of auditory and visual time intervals at common electrode locations (e.g. some of the parieto-occipital and parietal electrodes). However, at these common locations, the after-effects mainly occurred in distinct time windows and were in the opposite direction for each modality. Therefore, even for a common cortical site (e.g. PO8) at which significant auditory and visual time interval after-effects were observed, our results point to distinct characteristics and temporal dynamics for each modality.

\section{Sub-second time interval adaptation}

Our findings demonstrate for the first time that adapting to different time intervals significantly alters the ERPs elicited by visual motion (i.e. a visual feature dependent on the time interval between individual frames). By indicating specific scalp sites and ERP components, our findings here shed light on the nature of the cortical processes involved in time interval after-effects on motion perception. Previous psychophysical data have found significant influences of time interval adaptation on visual motion perception (Zhang et al., 2012). The after-effects of auditory and visual time intervals were in the same direction suggesting a role for a common timing mechanism. As opposed to the general view provided by these motion studies (see also Chen \& Zhou, 2014; Zhang \& Chen, 2016), we found distinct spatiotemporal characteristics of intramodal and cross-modal time interval after-effects. Accordingly, our ERP results and the follow-up behavioural findings on perceived speed emphasize the recruitment of distinct sensory processes rather than a central supramodal network for the adaptation after-effects on visual motion. By considering a more general argument in sub-second timing, we interpret our results as support for the distributed nature of the adaptation induced after-effects (Murai et al., 2016). Our results do not completely preclude a central supramodal mechanism and the possibility of deep brain structures being involved in timing computations. However, given both the lack of a cluster of electrodes commonly affected by the auditory and visual time intervals and the distinct changes in the spatiotemporal profile of the neural activity for each modality, our findings are consistent with the general notion also suggesting the involvement of distributed sites in subsecond timing. 


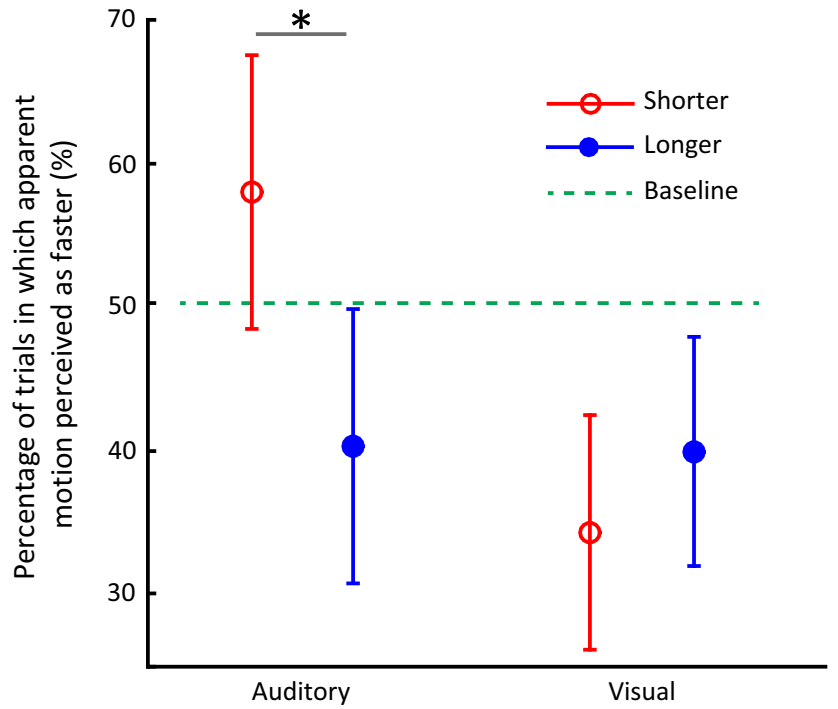

FIG. 8. Post-adaptation speed judgements $(N=8)$. The plot indicates the proportion of trials in which the apparent motion was judged to move faster than the drifting grating. The open and filled symbols correspond to shorter and longer time interval adaptations, respectively. The dotted line indicates the baseline $(50 \%)$ level. Error bars indicate standard error $( \pm$ SEM) across subjects. A significant difference between shorter and longer conditions was marked with an asterisk sign (paired $t$-test, ${ }^{*} P<0.05$ ). [Colour figure can be viewed at wileyonlinelibrary.com].

In general, compared with vision, audition has been found to have a dominant role in temporal processing and tasks dependent on timing (for a review see Chen \& Vroomen, 2013). Of particular interest is that learning studies revealed asymmetries between two modalities (Bratzke et al., 2012; Barakat et al., 2015; McGovern et al., 2016). For instance, Barakat et al. (2015) have recently reported that training observers in an auditory (but not visual) rhythm discrimination significantly improved their performance in a subsequent visual rhythm discrimination task. Similarly, using a more comprehensive design, McGovern et al. (2016) showed that transfer of perceptual learning between two modalities is specific to tasks and particular conditions. For a temporal discrimination task, training observers in the auditory domain transferred to the visual domain (i.e. decreased discrimination thresholds in vision), but not vice versa. Even though the adaptation phase in the current study was brief and did not include a specific learning paradigm, we also found dominant effects of auditory time interval adaptation on the evoked activity and perceived speed relative to visual time interval conditions. In this respect, our results are in line with the view supporting auditory dominance in tasks and/or stimulus attributes based on timing and time interval.

There has also been a growing interest to understand the basic principles underlying temporal recalibration between two modalities. Several studies have suggested that even passive exposure a single asynchronous audiovisual event can induce recalibration. The size of such rapid recalibration was found to be comparable to the one by prolonged periods of adaptation (Van der Burg et al., 2013; De Niear et al., 2017). A recent study by Simon et al. (2017) have identified ERP components and sites play an important role in these rapid after-effects. The changes associated with rapid recalibration were found to be over late ERP components and they were mostly centred over parietal and central regions. Interestingly, the identified cluster of electrodes were also similar to the ones reported here. To understand the progression of time interval after-effects in the present study, we compared averaged ERP amplitudes between early and late trials. Except for the 140- to 180-ms time range, we did not find a significant difference between these subset of trials. Therefore, similar to these recalibration studies, our findings suggest that for the time windows other than 140 - to 180 -ms time range the sub-second time interval after-effects may be efficiently induced at the initial phase of an adaptation block.

Contingent negative variation (CNV), an ERP component over fronto-central regions first described by Walter et al. (1964), has attracted great attention from researchers working on timing and time perception (for reviews see Van Rijn et al., 2011; Kononowicz \& Penney, 2016). Even though it is still subject to debate, some aspects of $\mathrm{CNV}$ have been found to be correlated with changes in perceptual timing (Macar et al., 1999; Pfeuty et al., 2008; $\mathrm{Ng}$ et al., 2011). Strong CNV activity and correlations with timing were mostly reported by studies using a task requiring observers to either compare the time intervals (or durations) of stimuli explicitly or reproduce the time interval (or duration) of a stimulus through a motor action. Of particular importance to the current study, Li et al. (2017) have recently shown that changes in CNV can also reflect the sub-second duration after-effects on the perceived duration of a subsequent event. During each trial, they first adapted observers to shorter or longer visual durations and then asked them to reproduce the duration of a visual test stimulus. Their results indicated a strong $\mathrm{CNV}$ component elicited by the visual test over fronto-central regions and this component was modulated by the duration used in the adaptation phase. Our results also indicated a negative component over frontal electrodes (Fig. 5). However, for both modalities, time interval adaptation did not yield a cluster of significant electrodes over these regions. Compared with the findings by Li et al. (2017), the CNV modulation was not apparent in our study. Here, instead of an explicit time interval discrimination or reproduction task, observers performed a motion direction discrimination. Considering that the link between $\mathrm{CNV}$ and behavioural performance depends on cognitive context, this apparent discrepancy between the two studies may be due to the different tasks observers engaged in.

\section{Auditory timing and visual motion}

It is well known that the temporal and spatial information provided by audition can affect visual motion perception (for reviews, see Soto-Faraco et al., 2003; Hidaka et al., 2015). For instance, the time interval between brief stationary sounds has been shown to alter the perception of apparent motion and speed (e.g. Getzmann, 2007; Kafaligonul \& Stoner, 2010, 2012). Although these behavioural studies suggest that auditory timing is actively used by the visual system to estimate motion, how auditory timing is involved in shaping motion perception and the corresponding neural activity are still unclear. Unlike this line of research, in the present study, the auditory clicks and the temporal information were not provided concurrently with visual motion. However, any significant change in the visual-evoked activity due to the auditory time interval used in the adaptation phase definitely gives us an indication of the cortical areas actively using auditory timing. Our results indicate that the significant differences between apparent motion ERPs for shorter and longer auditory adaptation are centred over parietal electrodes. The amplitudes of early components (50- to $80-\mathrm{ms}$ and 140 - to 180 ms time ranges) were significantly affected and the difference between two conditions extended over occipital scalp regions. The effects on the later $(>300 \mathrm{~ms})$ positive activity were more salient and mostly centred over right parieto-central electrodes. Building from these findings, we suggest that auditory timing may be 
progressively used for visual motion processing over these areas, starting from parieto-occipital up to parieto-central sites. Consistent with our observations here, visual motion areas and parietal cortices have been found to be involved in the sub-second time interval and duration percepts (Battelli et al., 2007; Salvioni et al., 2013; Shuler, 2016). The application of brain stimulation to right parietal cortices resulted in changes specific to sub-second duration judgements (Bueti et al., 2008; Dormal et al., 2016). In addition, a neuro-imaging study has shown that the activity in the right parietal cortex (i.e. inferior parietal lobe) can be modulated through duration adaptation (Hayashi et al., 2015). Multisensory studies also revealed significant audiovisual interactions over parietal regions. Multisensory integration effects between two modalities were observed on the neural activity over parieto-occipital, parietal and parieto-central sites (Molholm et al., 2002, 2006; Mercier et al., 2013). The amplitudes of N1 component (150- to 200-ms time range) over parietooccipital sites were found to be modulated by the timing of a brief sound and these modulations were suggested to correlate with the magnitude of the temporal ventriloquism elicited by an auditory and a visual event (Stekelenburg \& Vroomen, 2005). Therefore, the auditory time interval may take place for visual motion processing over these regions already known to be involved in both sub-second temporal processing and audiovisual interactions.

\section{Limitations and future directions}

In the current study, we used physically identical temporal intervals for both modalities. Although the difference between shorter and longer time interval conditions was perceptually obvious, they were not perceptually equated across modalities. As the temporal resolution of audition is superior to vision, we expect the difference between auditory time intervals to be more salient compared with visual time intervals. Such a difference may lead to dominant influences on ERPs through auditory adaptation. On the other hand, even though we identified significant sites and time windows for visual time intervals, our data and analysis may not reveal a more comprehensive and a detailed picture for visual adaptation conditions. We consider that this is possible, and characterizing the changes in ERP components over a variety of timescales including supra-second time intervals is important. Moreover, in the motion domain, manipulations on the duration of adaptation phase and the temporal delay between an adaptor and a test pattern have been found to be a fruitful approach to reveal different forms of adaptation and distinct temporal dynamics of sensory plasticity (e.g. Kanai \& Verstraten, 2005; Oluk et al., 2016). An interesting issue is to understand whether such different forms of time interval after-effects on apparent motion exist. Determining time interval after-effects for a broad range of timescales, adaptation durations and temporal delays awaits further ERP investigations.

\section{Conclusions}

Taken together, the present study demonstrates significant aftereffects of time interval adaptation on the ERPs elicited by visual apparent motion. We found that the changes in ERPs mainly occurred in different time windows and were mostly centred over distinct scalp sites for auditory and visual adaptors. Thus, our findings indicate distinct changes in the spatiotemporal profile of the neural activity for auditory and visual adaptation and suggest the involvement of distributed sensory processes in sub-second time interval adaptation. Moreover, compared with visual adaptors, the after-effects induced by auditory stimuli were more salient and in the opposite direction. In particular, these changes were mostly over right parietal electrodes. Accordingly, our findings here, in combination with accumulating evidence, also support the notion that neural structures in the right hemisphere play an important role in crossmodal temporal processing.

\section{Conflict of interest}

The authors declare no competing financial interests.

\section{Supporting Information}

Additional supporting information can be found in the online version of this article:

Fig. S1. Averaged ERP amplitudes within 140-180 ms time range for early and late trials.

Table S1. Results of paired $t$-tests comparing a specific adaptation condition with the no adaptation condition.

Table S2. Three-way repeated measures ANOvAs on the averaged ERPs estimated by using two different subset (early: first 10, late: last 10) of trials in an adaptation block.

\section{Acknowledgements}

We thank Mert Ozkan and Can Oluk for assistance in programming, data collection and analyses. We also thank Aaron Clarke and Jaap Munneke for discussions and comments on the manuscript. This work was supported by the Scientific and Technological Research Council of Turkey (TUBITAK Grant 113K547).

\section{Abbreviations}

ANOVA, analysis of variance; $\mathrm{CNV}$, contingent negative variation; ECG, electrocardiogram; EEG, electroencephalogram; ERP, event-related potential; ISI, inter-stimulus interval; PSE, point of subjective equality.

\section{Author contributions}

UK and HK designed and implemented Experiment 1. FZY and HK designed and implemented Experiment 2. UK and FZY collected and analysed the data. All authors interpreted the results and wrote the manuscript. All authors reviewed and approved the manuscript.

\section{Data accessibility}

The data used in the current study were collected under the provision of informed consent by the participants. Any access to the data will be granted in line with the informed consent and subject to the approval by the ethics committee.

\section{References}

Allen, P.J., Polizzi, G., Krakow, K., Fish, D.R. \& Lemieux, L. (1998) Identification of EEG events in the MR scanner: the problem of pulse artifact and a method for its subtraction. NeuroImage, 8, 229-239.

Barakat, B.K., Seitz, A.R. \& Shams, L. (2015) Visual rhythm perception improves through auditory, but not visual training. Curr. Biol., 25, 6061.

Battelli, L., Pascual-Leone, A. \& Cavanagh, P. (2007) The 'when' pathway of the right parietal lobe. Trends Cogn. Sci., 11, 204-210.

Brainard, D. (1997) The psychophysics toolbox. Spat. Vision, 10, 433436. 
Bratzke, D., Seifried, T. \& Ulrich, R. (2012) Perceptual learning in temporal discrimination: asymmetric cross-modal transfer from audition to vision. Exp. Brain Res., 221, 205-210.

Bruno, A. \& Cicchini, C.M. (2016) Multiple channels of visual time perception. Curr. Opin. Behav. Sci., 8, 131-139.

Bueti, D., Bahrami, B. \& Walsh, V. (2008) Sensory and association cortex in time perception. J. Cog. Neurosci., 20, 1054-1062.

Burr, D., Banks, M. \& Morrone, M. (2009) Auditory dominance over vision in the perception of interval duration. Exp. Brain Res., 198, 49-57.

Busse, L., Roberts, K.C., Crist, R.E., Weissman, D.H. \& Woldorff, M.G. (2005) The spread of attention across modalities and space in a multisensory object. P. Natl. Acad. Sci. USA, 102, 18751-18756.

Chen, L. \& Vroomen, J. (2013) Intersensory binding across space and time: a tutorial review. Atten. Percept. Psychophys., 75, 790-811.

Chen, L. \& Zhou, X. (2014) Fast transfer of crossmodal time interval training. Exp. Brain Res., 232, 1855-1864.

De Niear, M.A., Noel, J.-P. \& Wallace, M.T. (2017) The impact of feedback on the different time courses of multisensory temporal recalibration. Neural. Plast., 2017, 3478742.

Ding, Y., Martinez, A., Qu, Z. \& Hillyard, S.A. (2014) The earliest stages of visual cortical processing are not modified by attentional load. Hum. Brain Mapp., 35, 3008-3024.

Dormal, V., Javadi, A.H., Pesenti, M., Walsh, V. \& Cappelletti, M. (2016) Enhancing duration processing with parietal brain stimulation. Neuropsychologia, 85, 272-277.

Fendrich, R. \& Corballis, P.M. (2001) The temporal cross-capture of audition and vision. Percept. Psychophys., 63, 719-725.

Feng, W., Störmer, V.S., Martinez, A., McDonald, J.J. \& Hillyard, S.A. (2014) Sounds activate visual cortex and improve visual discrimination. $J$. Neurosci., 34, 9817-9824.

Freeman, E. \& Driver, J. (2008) Direction of visual apparent motion driven solely by timing of a static sound. Curr. Biol., 18, 1262-1266.

Getzmann, S. (2007) The effect of brief auditory stimuli on visual apparent motion. Perception, 36, 1089-1103.

Grahn, J.A., Henry, M.J. \& McAuley, J.D. (2011) FMRI investigation of cross-modal interactions in beat perception: audition primes vision, but not vice versa. NeuroImage, 54, 1231-1243.

Grondin, S. (2010) Timing and time perception: a review of recent behavioral and neuroscience findings and theoretical directions. Atten. Percept. Psychophys., 72, 561-582.

Guthrie, D. \& Buchwald, J.S. (1991) Significance testing of difference potentials. Psychophysiology, 28, 240-244.

Hayashi, M.J., Ditye, T., Harada, T., Hashiguchi, M., Sadato, N., Carlson, S., Walsh, V. \& Kanai, R. (2015) Time adaptation shows duration selectivity in the human parietal cortex. PLoS Biol., 13, e1002262.

Hidaka, S., Teramoto, W. \& Sugita, Y. (2015) Spatiotemporal processing in crossmodal interactions for perception of the external world: a review. Front. Integr. Neurosci., 9, 62.

Kafaligonul, H. \& Stoner, G.R. (2010) Auditory modulation of visual apparent motion with short spatial and temporal intervals. J. Vision, 10, 1-13.

Kafaligonul, H. \& Stoner, G.R. (2012) Static sound timing alters sensitivity to low-level visual motion. J. Vision, 12, 1-9.

Kanai, R. \& Verstraten, F.A.J. (2005) Perceptual manifestations of fast neural plasticity: motion priming, rapid motion aftereffect and perceptual sensitization. Vision. Res., 45, 3109-3116.

Kononowicz, T.W. \& Penney, T.B. (2016) The contingent negative variation (CNV): timing isn't everything. Curr. Opin. Behav. Sci., 8, 231237.

Levitan, C.A., Ban, Y.H.A., Stiles, N.R.B. \& Shimojo, S. (2015) Rate perception adapts across the senses: evidence for a unified timing mechanism. Sci. Rep., 5, 8857.

Li, B., Yuan, X. \& Huang, X. (2015) The aftereffect of perceived duration is contingent on auditory frequency but not visual orientation. Sci. Rep., 5, 10124.

Li, B., Chen, Y., Xiao, L., Liu, P. \& Huang, X. (2017) Duration adaptation modulates EEG correlates of subsequent temporal encoding. Neurolmage, 147, 143-151.

Macar, F., Vidal, F. \& Casini, L. (1999) The supplementary motor area in motor and sensory timing: evidence from slow brain potential changes. Exp. Brain Res., 125, 271-280.

Mauk, M.D. \& Buonomano, D.V. (2004) The neural basis of temporal processing. Annu. Rev. Neurosci., 27, 304-340.

McGovern, D.P., Astle, A.T., Clavin, S.L. \& Newell, F.N. (2016) Task-specific transfer of perceptual learning across sensory modalities. Curr. Biol., 26, R20-R21.
Merchant, H. \& de Lafuente, V. (2014) Introduction to the neurobiology of interval timing. Adv. Exp. Med. Biol., 829, 1-13.

Merchant, H., Harrington, D.L. \& Meck, W.H. (2013) Neural basis of the perception and estimation of time. Annu. Rev. Neurosci., 36, 313-336.

Mercier, M.R., Foxe, J.J., Fiebelkorn, I.C., Butler, J.S., Schwartz, T.H. \& Molholm, S. (2013) Auditory-driven phase reset in visual cortex: human electrocorticography reveals mechanisms of early multisensory integration. NeuroImage, 79, 19-29.

Molholm, S., Ritter, W., Murray, M.M., Javitt, D.C., Schroeder, C.E. \& Foxe, J.J. (2002) Multisensory auditory-visual interactions during early sensory processing in humans: a high density electrical mapping study. Brain Res. Cogn. Brain Res., 14, 115-128.

Molholm, S., Sehatpour, P., Mehta, A.D., Shpaner, M., Gomez-Ramirez, M., Ortigue, S., Dyke, J.P., Schwartz, T.H. et al. (2006) Audio-visual multisensory integration in superior parietal lobule revealed by human intracranial recordings. J. Neurophysiol., 96, 721-729.

Morein-Zamir, S., Soto-Faraco, S. \& Kingstone, A. (2003) Auditory capture of vision: examining temporal ventriloquism. Brain Res. Cogn. Brain Res., 17, 154-163.

Murai, Y., Whitaker, D. \& Yotsumoto, Y. (2016) The centralized and distributed nature of adaptation-induced misjudgments of time. Curr. Opin Behav. Sci., 8, 117-123.

Ng, K.K., Tobin, S. \& Penney, T.B. (2011) Temporal accumulation and decision processes in the duration bisection task revealed by contingent negative variation. Front. Integr. Neurosci., 5, 77.

Oluk, C., Pavan, A. \& Kafaligonul, H. (2016) Rapid motion adaptation reveals the temporal dynamics of spatiotemporal correlation between ON and OFF pathways. Sci. Rep., 6, 34073.

Oostenveld, R., Fries, P., Maris, E. \& Schoffelen, J.M. (2011) FieldTrip: open source software for advanced analysis of MEG, EEG, and invasive electrophysiological data. Comput. Intell. Neurosci., 2011, 156869.

Pelli, D. (1997) The VideoToolbox software for visual psychophysics: transforming numbers into movies. Spat. Vis., 10, 437-442.

Pfeuty, M., Ragot, R. \& Pouthas, V. (2008) Brain activity during interval timing depends on sensory structure. Brain Res., 1204, 112-117.

Picton, T.W., Bentin, S., Berg, P., Donchin, E., Hillyard, S.A., Johnson, R. Jr., Miller, G.A., Ritter, W. et al. (2000) Guidelines for using human event-related potentials to study cognition: recording standards and publication criteria. Psychophysiology, 37, 127-152.

Rammsayer, T.H., Borter, N. \& Troche, S.J. (2015) Visual-auditory differences in duration discrimination of intervals in the subsecond and second range. Front. Psychol., 6, 1626.

Recanzone, G.H. (2003) Auditory influences on visual temporal rate perception. J. Neurophysiol., 89, 1078-1093.

Salvioni, P., Murray, M.M., Kalmbach, L. \& Bueti, D. (2013) How the visual brain encodes and keeps track of time. J. Neurosci., 33, 12423-12429.

Senkowski, D., Saint-Amour, D., Kelly, S.P. \& Foxe, J.J. (2007) Multisensory processing of naturalistic objects in motion: a high-density electrical mapping and source estimation study. NeuroImage, 36, 877-888.

Shi, Z., Chen, L. \& Müller, H.J. (2010) Auditory temporal modulation of the visual Ternus effect: the influence of time interval. Exp. Brain Res., 203 723-735.

Shuler, M.G. (2016) Timing in the visual cortex and its investigation. Curr Opin. Behav. Sci., 8, 73-77.

Simon, D.M., Noel, J.-P. \& Wallace, M.T. (2017) Event related potentials index rapid recalibration to audiovisual temporal asynchrony. Front. Integr. Neurosci., 11, 8.

Soto-Faraco, S., Kingstone, A. \& Spence, C. (2003) Multisensory contributions to the perception of motion. Neuropsychologia, 41, 1847-1862.

Stekelenburg, J.J. \& Vroomen, J. (2005) An event-related potential investigation of the time-course of temporal ventriloquism. NeuroReport, 16, 641-644.

Van der Burg, E., Alais, D. \& Cass, J. (2013) Rapid recalibration to audiovisual asynchrony. J. Neurosci., 33, 14633-14637.

Van Rijn, H., Kononowicz, T.W., Meck, W.H., Ng, K.K. \& Penney, T.B. (2011) Contingent negative variation and its relation to time estimation: a theoretical evaluation. Front. Integr. Neurosci., 5, 91

Vroomen, J. \& Stekelenburg, J.J. (2010) Visual anticipatory information modulates multisensory interactions of artificial audiovisual stimuli. $J$. Cogn. Neurosci., 22, 1583-1596.

Walter, W.G., Cooper, R., Aldridge, V.J., McCallum, W.C. \& Winter, A.L. (1964) Contingent negative variation: an electric sign of sensorimotor association and expectancy in the human brain. Nature, 203, $380-384$.

Welch, R.B. \& Warren, D.H. (1980) Immediate perceptual response to intersensory discrepancy. Psychol. Bull., 88, 638-667. 
Wichmann, F.A. \& Hill, N.J. (2001a) The psychometric function: I. Fitting, sampling and goodness-of-fit. Percept. Psychophys., 63, 12931313.

Wichmann, F.A. \& Hill, N.J. (2001b) The psychometric function: II. Bootstrap-based confidence intervals and sampling. Percept. Psychophys., 63, $1314-1329$.
Zhang, Y. \& Chen, L. (2016) Crossmodal statistical binding of temporal information and stimuli properties recalibrates perception of visual apparent motion. Front. Psychol., 7, 434.

Zhang, H., Chen, L. \& Zhou, X. (2012) Adaptation to visual or auditory time intervals modulates the perception of visual apparent motion. Front. Integr. Neurosci., 6, 100. 